\title{
Changes of Inflammatory Mediators in Obese Patients After Laparoscopic Cholecystectomy
}

\author{
Gaetano Di Vita · Rosalia Patti · Fausto Famà · \\ Carmela Rita Balistreri · Giuseppina Candore • \\ Calogero Caruso
}

Published online: 18 May 2010

(C) Société Internationale de Chirurgie 2010

\begin{abstract}
Background Obesity is associated with the impairment of immunological functions. The aim of this study was to analyze some inflammatory mediators in obese subjects who underwent laparoscopic cholecystectomy.

Methods Seventeen consecutive female patients with a BMI ranging from 35 to $45 \mathrm{~kg} / \mathrm{m}^{2}$ (obese) and 17 consecutive female patients with BMI ranging from 20 to $25 \mathrm{~kg} / \mathrm{m}^{2}$ (nonobese) were included in the study. All patients were affected by symptomatic gallbladder stone disease and underwent laparoscopic cholecystectomy. Changes in levels of leukocytes, neutrophils, IL-6, IL-10, leptin, and adiponectin were evaluated.

Results We observed a significant increase in leukocyte and neutrophil levels in the obese subjects compared to the nonobese subjects. The serum levels of leptin and IL-6 were higher in the postoperative period (compared to the baseline values in both groups), and always higher in the obese. Both adiponectin and IL-10 increased in the postoperative period in nonobese subjects and was always higher than in the obese.

Conclusions Obese patients have a stronger acute inflammatory response than do nonobese subjects in reaction to surgical stress.
\end{abstract}

G. D. Vita $(\bowtie) \cdot$ R. Patti $\cdot$ F. Famà

General Surgery Unit, Department of Surgical and Oncological

Science, University of Palermo, Via Liborio Giuffrè n ${ }^{\circ} 5$,

Palermo, Italy

e-mail: divitagaetano@libero.it

C. R. Balistreri - G. Candore - C. Caruso

Immunosenescence Unit, Department of Pathobiology and

Biomedical Methodologies, University of Palermo,

Palermo, Italy

\section{Introduction}

Obesity is a pathological condition characterized by excessive fat deposits compared to the expected values for a given status, gender, and age and is often estimated by a body mass index (BMI) greater than 30 [1]. Obesity is a worldwide epidemic problem with significant adverse effects on public health and health-care costs. Its prevalence is increasing in all industrialized countries. It is the second leading cause of death in the U.S., with approximately 300,000 deaths per year and an estimated total annual health cost of $\$ 117$ billion [2]. Recent data from the U.S. National Center for Health Statistics reveal that $30 \%$ of adults over the age of 20 are obese, approximately 60 million people. The situation in Europe is becoming similar [1].

The disability and shortened life span associated with obesity are consequences of comorbidity conditions, including diabetes, hypertension, coronary heart disease, respiratory dysfunction, cancer, and impairment of immunological functions that are accompanied by a higher rate of infections and increased risk of delayed wound healing $[3,4]$, with a lower quality of life [5]. Furthermore, obesity is also thought to be a major risk factor for postoperative complications [6]. Some studies [6] suggested that obese patients have a high risk of infectious complications after surgery compared to nonobese patients; other studies [11] reported no correlation.

Although the pathogenesis of these conditions is multifactorial and to date not clearly defined, it is generally accepted that it is related, at least in part, to the metabolic activity of adipose tissue acting via elevated levels of inflammatory factors [14]. Adipose tissue is not only stored energy, it is involved in a number of functions as an endocrine organ [15]. It is known to be able to stimulate the production of some inflammatory markers, including 
C-reactive protein (CRP) and fibrinogen, through the direct production of proinflammatory cytokines such as interleukin (IL)-6 [16]. Some proteins such as leptin and adiponectin have been shown to play an important role in regulating immune function [17].

Taking into account the above considerations, the aim of this study was to analyze some immune-inflammatory parameters in obese patients who underwent surgery to explain (on the basis of biological results) the major incidence of postoperative infectious complications observed in these subjects. To avoid confounding effects linked to different diseases, as a surgical procedure model we chose the response to laparoscopic cholecystectomy (LC) for benign gallbladder disease. As we previous attested [18, 19], it is known that simple LC induces changes of some inflammatory mediators. As an expression of the inflammatory mediators, we measured changes in the levels of leukocytes, neutrophils, IL-6, IL-10, leptin, and adiponectin.

\section{Materials and methods}

Seventeen consecutive female patients with a BMI between 35 and $45 \mathrm{~kg} / \mathrm{m}^{2}$ and 17 consecutive female patients with a BMI between 20 and $25 \mathrm{~kg} / \mathrm{m}^{2}$ were enrolled in this study. All patients were admitted to the Surgery Unit of the Surgical Department of the University of Palermo from April 2005 to June 2007, and they were affected by symptomatic and uncomplicated gallbladder stone disease. Exclusion criteria were the presence of diabetes or endocrine, hepatic, renal, neoplastic, inflammatory, or immunological disease. Furthermore, patients who used drugs that could influence the inflammatory response such as steroid or nonsteroidal anti-inflammatory drugs and immunomodulant agents were excluded. The study was approved by the Hospital Ethics Committee and all patients gave written informed consent. Patients were divided into two groups according to their BMI. The first group included patients with $\mathrm{BMI}=35$ $45 \mathrm{~kg} / \mathrm{m}^{2}$ (obese group) and the second group included patients with $\mathrm{BMI}=20-25 \mathrm{~kg} / \mathrm{m}^{2}$ (nonobese group). Preoperatively, all patients received antibiotic prophylaxis with $1 \mathrm{~g}$ of intramuscular cefuroxime and antithrombotic prophylaxis using low-molecular-weight eparine. All subjects underwent the standard anesthetic procedures, using thiopental sodium for induction, vecuronium for neuromuscular block, and isofluorane and fentanyl citrate for analgesia. All patients underwent a laparoscopic cholecystectomy (LC). All the operations were carried out by the same senior surgeon (GD) with long experience in laparoscopic surgery. The Anglo-Saxon technique with four trocars was preferred, with a $\mathrm{CO}_{2}$ pneumoperitoneum maintained at $12-14 \mathrm{mmHg}$. In all patients the telescope trocar was introduced with the open technique through a small sovra-umbilical incision using a special endotip trocar. An analgesic (not an anti-inflammatory drug) was given as postoperative analgesia as requested. Postoperative pain, analgesic consumption, length of hospital stay, and postoperative complications were recorded. Pain was assessed twice daily for the first week after surgery using a linear analog scale ranging from 0 (no pain) to 10 (worst conceivable pain) [20]. Peripheral venous blood samples were collected $24 \mathrm{~h}$ before surgery and then 6,24 , and $48 \mathrm{~h}$ after surgery.

Absolute numbers and percentages of leukocyte blood counts were determined using ADVIA 120 (Bayer Diagnostics, Munich, Germany). Sera were obtained from blood within $30 \mathrm{~min}$ of venipuncture by clotting and centrifugation at $400 \times g$ for $10 \mathrm{~min}$. Samples were aliquoted and frozen at $-70^{\circ} \mathrm{C}$ until assay. For quantitative determination of the cytokines and adipokines (IL-6, IL-10, leptin, and adiponectin), assays were performed with commercially available kits according to the manufacturer's instructions (R\&D Systems Inc., Minneapolis, MN, USA). To standardize our results, in all the assays cytokine and adipokine reference preparations were tested. These assays employed the quantitative sandwich enzyme immune assay technique, characterized by use of monoclonal antibodies specific for these molecules, precoated onto a solid phase. All the standards and samples were tested in duplicate. By using these kits, the detection limits were $7.8 \mathrm{pg} / \mathrm{ml}$ for leptin, $0.246 \mathrm{ng} / \mathrm{ml}$ for adiponectin, $3.9 \mathrm{pg} / \mathrm{ml}$ for IL-10, and $0.7 \mathrm{pg} / \mathrm{ml}$ for IL-6.

A sample-size calculation estimated that 17 patients would be required in each arm to show 1 standard deviation difference of all parameters evaluated between the obese and nonobese groups with a power of 0.8 and alpha level of 0.05 . Values are given as mean $\pm \mathrm{SD}$. An unpaired $t$ test with Welch correction was used to compare the differences in the characteristics of the two patient groups. One-way analysis of variance (ANOVA, corrected by Bonferroni) was used to evaluate significant differences in the levels of cytokines and adipokines between the two groups. Differences were considered significant when $p<0.05$. Furthermore, in both groups, postoperative values were compared to baseline values by ANOVA (corrected by Bonferroni).

\section{Results}

Patient characteristics

Age, gender, anesthesiology grading (ASA), operative time, length of hospital stay, BMI, and comorbidities are given in Table 1 . The only characteristic with a significant difference between the two groups was BMI. 
Table 1 Characteristics of the patients in the two study groups

$\chi^{2}$ test was used to compare the continuous variables and unpaired $t$ test with Welch correction was used to compare the differences between the two groups

\begin{tabular}{lllr}
\hline & $\begin{array}{l}\text { Obese group } \\
(n=17)\end{array}$ & $\begin{array}{l}\text { Nonobese group } \\
(n=17)\end{array}$ & $p$ value \\
\hline Age $[$ median (range)] & $44.5(20-65)$ & $45.0(23-67)$ & 0.9315 \\
BMI $\left(\mathrm{kg} / \mathrm{m}^{2}\right)$ [median (range)] & $43.0(35-45)$ & $22(20-25)$ & $<0.0001$ \\
ASA & & & \\
I & 3 & 3 & 0.9668 \\
II & 9 & 7 & 0.8538 \\
III & 4 & 6 & 0.6303 \\
IV & 1 & 1 & 0.9668 \\
Duration of operation $(\min )($ mean $\pm \mathrm{SD})$ & $76.2 \pm 20.1$ & $73.8 \pm 17.6$ & 0.7101 \\
Hospital stay $(\mathrm{h})($ mean $\pm \mathrm{SD})$ & $50.4 \pm 15.3$ & $54.1 \pm 13.7$ & 0.4573 \\
Hypertension & 1 & 1 & 0.9668 \\
\hline
\end{tabular}

\section{Clinical outcomes}

No significant differences were detected between the two groups with respect to postoperative pain as measured on a visual analog scale at each time point and analgesic use (data not shown). Body temperature changes were similar in both groups, peaking at $24 \mathrm{~h}$ after surgery and returning to preoperative levels $48 \mathrm{~h}$ after the operation. The patients of both groups were discharged within $72 \mathrm{~h}$ after the LC. All patients had an uncomplicated postoperative course. No patient in either group developed a wound, respiratory, or urinary tract infection or other morbidity in the postoperative period. There was no need for reoperation or blood transfusion in either group.

\section{Leukocyte and neutrophil counts}

In both groups of patients the postoperative values of leukocytes and neutrophils were, on the whole, significantly higher compared to their baseline values (Table 2). There was a significant increase in leukocyte and neutrophil levels preoperatively and at 6,24 , and $48 \mathrm{~h}$ after surgery in obese patients compared to nonobese patients (Table 2).
IL-6, leptin, IL-10, and adiponectin

Data on proinflammatory cytokines IL-6, IL-10, leptin, and adiponectin are presented in Fig. 1a-d, respectively. In general, the serum levels of both leptin and IL-6 increased in the postoperative period compared to their baseline values. In particular, the IL-6 level significantly increased in the obese subjects 24 and $48 \mathrm{~h}$ after surgery, whereas in the nonobese subjects a significant increase was observed only $48 \mathrm{~h}$ after surgery. Interestingly, the IL- 6 values of obese patients were always higher than those in nonobese subjects at all times (Fig. 1a). The serum levels of leptin were significantly higher in obese patients 24 and $48 \mathrm{~h}$ postoperatively compared to those of nonobese subjects at any time point. A comparison between the two groups showed higher values of leptin in the obese patients compared to the nonobese subjects (Fig. 1c).

Overall, the serum levels of both adiponectin and IL-10 were higher in the postoperative period in the nonobese patients, as seen in Fig. $1 \mathrm{~b}$ and d. In particular, IL-10 was significantly higher in nonobese patients 24 and $48 \mathrm{~h}$ after cholecystectomy, whereas in the obese group its levels showed a significant increase only at $24 \mathrm{~h}$ after surgery. IL-10 levels of nonobese subjects were also higher than

Table 2 Leukocyte and neutrophil counts in the two study groups

\begin{tabular}{|c|c|c|c|c|c|c|c|c|}
\hline & \multicolumn{2}{|l|}{ Pre } & \multicolumn{2}{|l|}{ 6th $\mathrm{h}$} & \multicolumn{2}{|l|}{24 th $\mathrm{h}$} & \multicolumn{2}{|l|}{ 48th h } \\
\hline & Obese & Nonobese & Obese & Nonobese & Obese & Nonobese & Obese & Nonobese \\
\hline Leukocytes (absolute number) & $9.9(2.5)^{\mathrm{a}}$ & $6.4(1.1)$ & $15.2(2.8)^{\mathrm{a}, *}$ & $8.3(2.4)^{\dagger}$ & $14.6(3.0)^{\mathrm{a}, *}$ & $10.4(2.6)^{\dagger}$ & $14.5(2.6)^{\mathrm{a}, *}$ & $10.1(2.3)^{\dagger}$ \\
\hline Neutrophils (absolute number) & $7.3(1.2)^{\mathrm{a}}$ & $4.5(1.1)$ & $12.9(2.3)^{\mathrm{a}}$ & $7.2(1.0)^{\dagger}$ & $12.0(1.9)^{\mathrm{a}}$ & $8.3(1.2)^{\dagger}$ & $11.3(1.0)^{\mathrm{a}}$ & $7.5(1.0)^{\dagger}$ \\
\hline
\end{tabular}

Values are mean (standard deviation)

${ }^{a}$ Comparison by unpaired $t$ test with Welch correction of values of leukocytes and neutrophils of obese and nonobese patients at preop, 6, 24 and $48 \mathrm{~h}$. A significance of $p<0.0001$ was obtained for both parameters. Within each group, one-way analysis of variance by ANOVA with Bonferroni correction was used to analyze the differences for each time point compared to preoperative values

* Pre vs. 6: $p<0.001$; pre vs. $24, p<0.001$; pre vs. $48, p<0.001$ for obese group

$\dagger$ Pre vs. $6, p<0.001$; pre vs. $24, p<0.001$; pre vs. $48, p<0.001$ for nonobese group for both parameters 


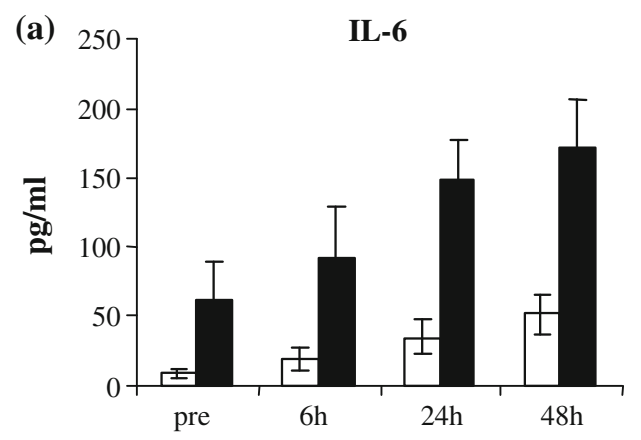

(c)

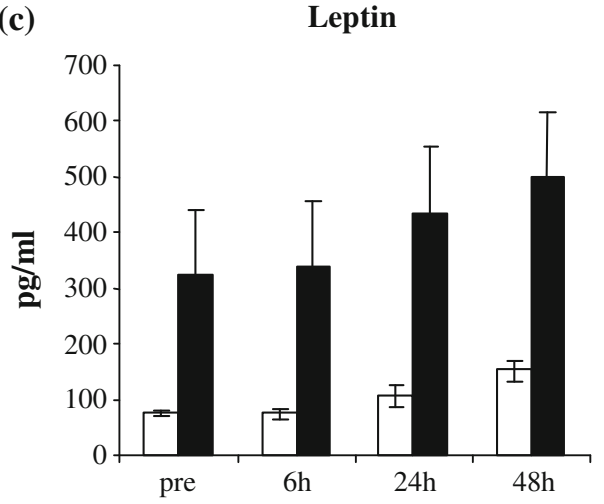

Fig. 1 a Pre- and postoperative IL-6 serum levels in obese (black bar) and nonobese (white bar) patients. Concerning IL-6, differences between baseline and postoperative values were observed only at $48 \mathrm{~h}$ $(p<0.001$ by ANOVA test with Bonferroni's correction) in nonobese patients. In obese patients, the differences between baseline and postoperative values were significant at 24 and $48 \mathrm{~h}(p<0.01$ and $p<0.001$, respectively, by ANOVA test with Bonferroni's correction). In addition, significance was achieved comparing values of nonobese and obese patients at all times after operation $(p<0.001$ by ANOVA test with Bonferroni's correction). b Pre- and postoperative IL-10 serum levels in obese (black bar) and nonobese (white bar) patients. A significant increase between baseline and postoperative values was observed at 24 and $48 \mathrm{~h}(p<0.001$ and $p<0.001$, respectively, by ANOVA test with Bonferroni's correction) in nonobese patients. In obese patients, the differences between baseline and postoperative values were significant only at $24 \mathrm{~h}(p<0.01$ by ANOVA test with Bonferroni's correction). In addition, significance was achieved comparing values of nonobese and obese patients at 24 and $48 \mathrm{~h}$ after operation $(p<0.01$ and $p<0.001$, respectively, by ANOVA test with Bonferroni's correction). c Pre- and postoperative

IL-10 levels of obese patients, achieving statistical significance at 24 and $48 \mathrm{~h}$ after operation (Fig. 1b).

Similarly, the serum levels of adiponectin reached a significant increase postoperatively at all time points only in nonobese patients. In addition, a significant difference in adiponectin values was found between obese and nonobese patients at each point examined (Fig. 1d).

\section{Discussion}

In this study, increased levels of proinflammatory markers, including leptin and IL-6, were detected in the blood of
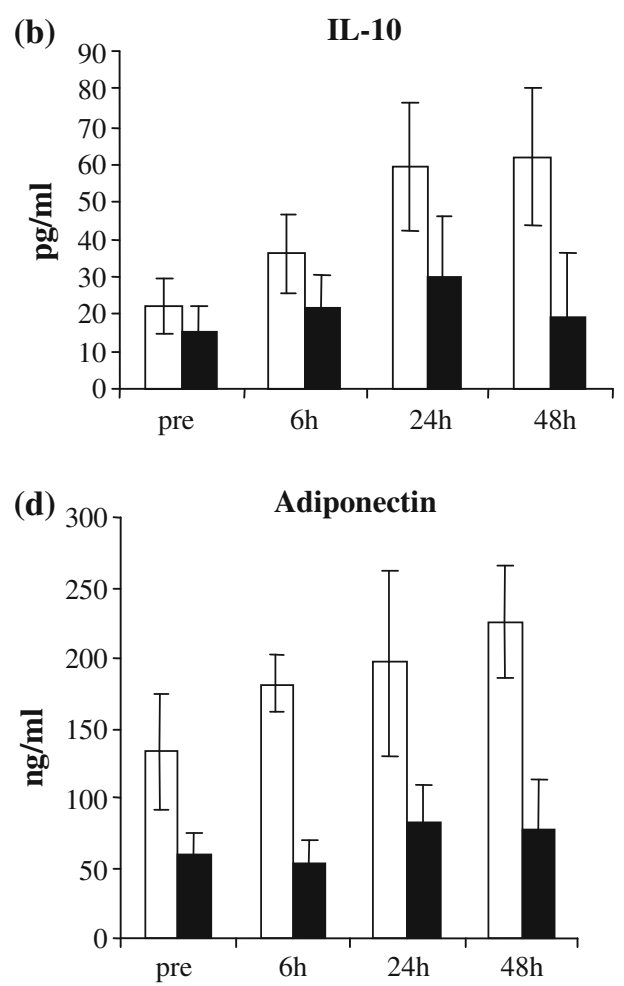

leptin serum levels in obese (black bar) and nonobese (white bar) patients. A significant increase between baseline and postoperative values was observed only at 24 and $48 \mathrm{~h}(p<0.05$ and $p<0.001$, respectively, by ANOVA test with Bonferroni's correction) in obese patients. In nonobese patients, the leptin postoperative values were not significantly higher when compared to baseline values. In addition, significance was obtained comparing values of nonobese and obese patients at all times after operation $(p<0.01, p<0.001$, $p<0.001$, and $p<0.0001$, respectively, by ANOVA test with Bonferroni's correction) d Pre- and postoperative adiponectin serum levels in obese (black bar) and nonobese (white bar) patients. A significant increase in adiponectin postoperative values compared to the baseline values was observed only in nonobese patients at 6,24 , and $48 \mathrm{~h}(p<0.05, p<0.01$, and $p<0.001$, respectively, by ANOVA test with Bonferroni's correction). In obese patients the adiponectin postoperative values were not significantly higher compared to baseline values. In addition, significance was found when the values of nonobese and obese patients at all times after operation were compared $(p<0.001$ by ANOVA test with Bonferroni's correction)

obese patients compared to nonobese individuals after LC. On the other hand, levels of IL-10 and adiponectin were lower in obese subjects compared to nonobese individuals. The latter findings suggest that in nonobese subjects, the inflammatory response following LC is attenuated by the inhibitory activity of these anti-inflammatory cytokines. All these data are in agreement with the evidence that obesity is characterized by low-grade systemic inflammation $[16,21]$.

It is commonly assumed that obese patients have a higher risk of surgical complications than do normal subjects [7]. A few previous studies that evaluated the 
impact of obesity on perioperative outcome produced questionable results [7]. Bangbade et al. [6], in their retrospective review of 7271 cases of postoperative complications after major or moderate noncardiac surgery, showed that obese patients had a higher prevalence of myocardial infarction, peripheral nerve injury, wound infection, and urinary tract infection, with a mortality rate of $2.2 \%$ compared to $1.2 \%$ for normal subjects [6]. Dindo et al. [7] investigated how obesity affected outcome of elective general surgery in a cohort of more than 6000 patients followed prospectively over 10 years. They suggested that obese patients face a higher risk for surgical site infections after open surgery, probably related to the presence of excessive fat tissue which, in their opinion, may predispose the patient to impaired wound healing and infections in the presence of concomitant factors such as hematoma [7]. Furthermore, Tanaka et al. [8] showed that obese patients have an underlying immune impairment in lymphocyte responsiveness which might contribute to higher rates of wound infections [8]. More recently, data collected from a review examining the impact of obesity on postoperative morbidity after colorectal surgery [9] suggested that obesity represents a significant risk factor for wound infections, anastomotic leakage, and pulmonary and thromboembolic problems [9]. Accordingly, in a multivariate analysis of 608 patients who underwent digestive tract surgery, subjects with BMI $>30$ had an adjusted odds ratio (OR) for surgical site infections of 4.8 [10]. Conversely, a retrospective review by Ondrula et al. [11] of 960 colorectal procedures performed during a 5-year period found that obesity was not significantly associated with wound infections [11]. Also, similar results have been observed after cardiac surgery [12]. Finally, Mullen et al. [13] even suggested that after nonbariatric general surgery, overweight and obese patients may paradoxically have better outcomes than normalweight patients.

Recently, both clinical and experimental studies showed that obesity produced immune response impairment $[4,8$, 22]. In particular, Chandra and $\mathrm{Au}$ [22] found that $38 \%$ of obese children showed variable impairment of cell-mediated immune responses such as delayed cutaneous hypersensitivity, abnormal lymphoproliferative response to mitogens, and a reduction in intracellular bacteria-killing capacity by polymorphonuclear leukocytes. Some reports have also linked obesity to a lower production of antibodies after hepatitis B vaccination [23].

The current view of adipose tissue is that it is an active secretor organ able to produce immune-related proteins, including cytokines, adipokines, fibrinogen, and acute phase proteins such as CRP [16]. Leptin is a cytokine that has a fundamental role in the regulation of food intake and energy balance [16], with a crucial function in regulating immunity [24]. In particular, leptin protects T lymphocytes from apoptosis and regulates T-cell proliferation and activation [21]. It also influences cytokine production from $\mathrm{T}$ lymphocytes, monocyte activation, and phagocytosis [21]. Thus, leptin acts as a proinflammatory cytokine and its circulating levels directly correlate with adipose tissue mass [25]. On the other hand, adiponectin, although released by adipocytes, does not increase with obesity; its levels are reduced in obese subjects [22]. Adiponectin acts as an anti-inflammatory by inhibiting IL-6 production accompanied by induction of the anti-inflammatory cytokines IL-10 and IL-1 receptor antagonist [26].

Our results indicate that obese patients have a stronger acute inflammatory response than do nonobese subjects after surgical stress. The severity of surgical stress varies in accordance with the host response after surgical procedures. Proinflammatory cytokines are thought to mediate surgical stress, with neurohumoral response assumed to be regulated by the production of cytokines [27]. The complex cascade of acute-phase response, regulated by an adequate balance of inflammatory mediators, is also designed to isolate and destroy microbial pathogens, to activate tissue repair processes, and to facilitate a return to physiological homeostasis [28]. Therefore, when there is hyperactivity in the acute-phase response due to an increase in the inflammatory mediators, as we observed in obese patients, there is an elevated risk of immune impairment that could be responsible for an increase in postoperative complications [27, 29].

The clinical significance of our results remains unclear because our study is only observational. Hence, we cannot conclude that there is a causal relationship between the cytokines leptin and adiponectin and acute-phase response and the increased susceptibility to postoperative complications observed in obese patients. However, it must be taken into account that it has been claimed that proinflammatory mediators as well as cytokines and leptin play an important role in the pathogenesis of postoperative complications [30].

\section{References}

1. World Health Organization (1998) Obesity: preventing and managing the global epidemic. WHO, Geneva

2. Mokdad AH, Marks JS, Stroup DF et al (2004) Actual causes of death in the United States, 2000. JAMA 291:1238-1245

3. Khan LK, Bowman BA (1999) Obesity: a major global public health problem. Annu Rev Nutr 19:13-17

4. Martí A, Marcos A, Martínez JA (2001) Obesity and immune function relationships. Obes Rev 2:131-140

5. Livingstone EH, Ko CY (2002) Use of the health and activities limitation index as a measure of quality of life in obesity. Obes Res 10:824-832

6. Bamgbade OA, Rutter TW, Nafiu OO et al (2007) Postoperative complications in obese and nonobese patients. World J Surg $31: 556-560$ 
7. Dindo D, Muller MK, Weber M et al (2003) Obesity in general elective surgery. Lancet 361:2032-2035

8. Tanaka S, Inoue S, Isoda F et al (1993) Impaired immunity in obesity: suppressed but reversible lymphocyte responsiveness. Int J Obes Relat Metab Disord 17:631-636

9. Gendall KA, Raniga S, Kennedy R et al (2007) The impact of obesity on outcome after major colorectal surgery. Dis Colon Rectum 50:2223-2237

10. de Oliveira AC, Ciosak SI, Ferraz EM et al (2006) Surgical site infection in patients submitted to digestive surgery: risk prediction and the NNIS risk index. Am J Infect Control 34:201-207

11. Ondrula DP, Nelson RL, Prasad ML et al (1992) Multifactorial index of preoperative risk factors in colon resections. Dis Colon Rectum 35:117-122

12. Brandt M, Harder K, Walluscheck KP et al (2001) Severe obesity does not adversely affect perioperative mortality and morbidity in coronary artery bypass surgery. Eur J Cardiothorac Surg 19:662666

13. Mullen JT, Moorman DW, Davenport DL (2009) The obesity paradox: body mass index and outcomes in patients undergoing nonbariatric general surgery. Ann Surg 250:166-172

14. Schwarzenberg SJ, Sinaiko AR (2006) Obesity and inflammation in children. Paediatr Respir Rev 7:239-246

15. Gregoire FM, Smas CM, Sul HS (1998) Understanding adipocyte differentiation. Physiol Rev 78:783-809

16. Cancello R, Clement K (2006) Is obesity an inflammatory illness? Role of low-grade inflammation and macrophage infiltration in human white adipose tissue. BJOG 113:1141-1147

17. O'Rourke RW, Kay T, Scholz MH et al (2005) Alterations in T-cell subset frequency in peripheral blood in obesity. Obes Surg 15:1463-1468

18. Di Vita G, Sciumè C, Milano S et al (2001) Inflammatory response in open and laparoscopic cholecystectomy. Ann Ital Chir 72:669-673

19. Schietroma M, Carlei F, Cappelli S et al (2007) Effects of cholecystectomy (laparoscopic versus open) on PMN-elastase. Hepatogastroenterology 54:342-345
20. Huskisson EC (1982) Measurement of pain. J Rheumatol 9:768769

21. Fantuzzi G (2005) Adipose tissue, adipokines, and inflammation. J Allergy Clin Immunol 115:911-919

22. Chandra RK, Au B (1980) Spleen hemolytic plaque-forming cell response and generation of cytotoxic cells in genetically obese (C57Bl/6J ob/ob) mice. Int Arch Allergy Appl Immunol 62:9498

23. Weber DJ, Rutala WA, Samsa GP et al (1986) Impaired immunogenicity of hepatitis B vaccine in obese persons. N Engl J Med 314:1393

24. Lago F, Dieguez C, Gómez-Reino J et al (2007) Adipokines as emerging mediators of immune response and inflammation. Nat Clin Pract Rheumatol 3:716-724

25. Maffei M, Fei H, Lee GH et al (1995) Increased expression in adipocytes of ob RNA in mice with lesions of the hypothalamus and with mutations at the db locus. Proc Natl Acad Sci USA 92:6957-6960

26. Wolf AM, Wolf D, Rumpold H et al (2004) Wolf adiponectin induces the anti-inflammatory cytokines IL-10 and IL-1RA in human leukocytes. Biochem Biophys Res Commun 323:630-635

27. Angele MK, Faist E (2002) Clinical review: immunodepression in the surgical patient and increased susceptibility to infection. Crit Care 6:298-305

28. Di Vita G, Patti R, Buscemi S et al (2006) Acute phase response in oldest-old individuals after surgical stress. J Am Geriatr Soc 54:561-563

29. Sietses C, Beelen RH, Meijer S et al (1999) Immunological consequences of laparoscopic surgery, speculations on the cause and clinical implications. Langenbecks Arch Surg 384:250-258

30. Bone RC (1996) Towards a theory regarding the pathogenesis of the systemic inflammatory response syndrome: what we do and do not know about cytokine regulation. Crit Care Med 24:163172 